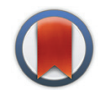

CrossMark

Cite this: Food Funct., 2016, 7, 3637

DOI: $10.1039 /$ c6fo90026g

www.rsc.org/foodfunction

\section{Correction: The anti-inflammatory effect of a glycosylation product derived from the high hydrostatic pressure enzymatic hydrolysate of a flatfish byproduct}

\author{
In-Hu Choe, ${ }^{a}$ Hyeon Jin Jeon, ${ }^{b}$ Sung-Hwan Eom, ${ }^{b}$ Young-Ki Han, ${ }^{b}$ \\ Yoon Sook Kim ${ }^{{ }^{b}}$ and Sang-Hoon Lee ${ }^{\star a, b}$
}

Correction for 'The anti-inflammatory effect of a glycosylation product derived from the high hydrostatic pressure enzymatic hydrolysate of a flatfish byproduct' by In-Hu Choe, et al., Food Funct., 2016, 7, 2557-2565

The authors regret the omission of the Acknowledgements section from the original manuscript, and wish to add the following text:

This research was a part of the project titled 'Development of allergen reduced FPH with ultra high pressure and Maillard reaction', funded by the Ministry of Oceans and Fisheries, Korea.

The Royal Society of Chemistry apologises for these errors and any consequent inconvenience to authors and readers.

${ }^{a}$ Department of Food Biotechnology, University of Science and Technology, Daejeon 305-350, South Korea

${ }^{b}$ Korea Food Research Institute, Baekhyun-dong, Seongnam, Gyeonggi 463-746, South Korea. E-mail: shnlee@kfri.re.kr; Fax: +82-31-780-9281; Tel: +82-31-780-9281 